\title{
Detección de Mastitis Subclínica Bovina y factores asociados, en fincas lecheras de la Provincia del Cañar - Biblián, Ecuador
}

\author{
Detection of Subclinic Bovine Mastitis and associated factors, in dairy farms of the Province \\ of Cañar - Biblián, Ecuador
}

\author{
Mercy Cuenca-Condoy ${ }^{1,2}$ iD, Dionel García-Bracho' ${ }^{\text {(D) , Lourdes Reinoso-García }}{ }^{\text {(D) , Juan González-Rojas }}{ }^{3}$ iD y \\ José Torracchi-Carrasco ${ }^{3}$ iD

\begin{abstract}
${ }^{1}$ Universidad del Zulia, Facultad de Ciencias Veterinarias. Maracaibo, Venezuela. ${ }^{2}$ Universidad Católica de Cuenca, Carrera de Medicina Veterinaria. Cuenca, Ecuador. ${ }^{3}$ Centro de Investigación, Innovación y Transferencia de Tecnología (CIITT), Universidad Católica de

Cuenca. Cuenca, Ecuador. Correo electrónico: mccuencac@ucacue.edu.ec
\end{abstract}

\section{RESUMEN}

El objetivo del estudió fue detectar la presencia de Mastitis Subclínica Bovina (MSB) y factores asociados a la enfermedad, en 23 fincas lecheras de la provincia del Cañar, parroquia Biblián, Ecuador, que se encuentran conformadas por rangos de 5 a 29 vacas en producción lechera; se evaluaron 360 vacas (1.440 cuartos mamarios), de las cuales se tomaron muestras de leche y se realizó la prueba de campo California Mastitis Test (CMT) verificando la categoría de mastitis subclínica (MS). Las muestras de leche positivas a CMT fueron recolectadas en frascos estériles por duplicado y transportadas al laboratorio conservando la cadena de frío para realizar el conteo de células somáticas (CCS). Los factores asociados a MS fueron tipo de ordeño y prácticas de manejo. Los resultados determinaron una prevalencia de $9,1 \%$ de MSB en la zona de estudio y de $56,5 \%$ a nivel de hatos ganaderos. En cuanto a factores asociados se encontró que el ordeño mecánico y la aplicación de algunas prácticas, como el secado de los pezones con toalla no individual, incrementa el riesgo en las vacas de padecer la enfermedad. Se concluye que la prevalencia de MSB a nivel de hatos ganaderos es alta y que el secado de pezones con toalla colectiva incrementa el riesgo de padecer la enfermedad.

Palabras clave: Mastitis; prácticas de manejo; factores de riesgo

\begin{abstract}
The objective of the study was to detect the presence of Subclinical Bovine Mastitis (SBM) and factors associated with the disease in 23 dairy farms in the Province of Cañar, Biblián Parish, Ecuador, comprised by a range of 5 to 29 cows in milk production; the samples were taken from 360 cows (1,440 mammary quarters) and the California Mastitis Test (CMT) was performed to verify the category of subclinical mastitis (SCM). The CMT-positive milk samples were collected in sterile bottles in duplicate and transported to the laboratory preserving the cold chain for somatic cell count (SCC). Factors associated with Subclinical Bovine Mastitis (SBM) were milking type and management practices. The results determined a prevalence of $9.1 \%$ of SBM in the study area and $56.5 \%$ at the herd level. In terms of associated factors, it was found that mechanical milking and the application of some practices, such as drying teats with a non-individual towel, increase the risk of cows suffering from the disease. It is concluded that the prevalence of MSB at the herd level is high and that teat drying with a collective towel increases the risk of having the disease.
\end{abstract}

Key words: Mastitis; prevalence; risk factors 


\section{INTRODUCCIÓN}

En Ecuador, dentro del ámbito ganadero predomina la cría de vacunos, con un total de 4,3 millones (Mill) de cabezas a nivel nacional, alcanzando en el 2019 una producción de 6,65 Mill litros $\cdot \mathrm{dia}^{-1}\left(\mathrm{~L} \cdot \mathrm{d}^{-1}\right)$ de leche, aportando la región Sierra con 5,16 Mill de $L \cdot d^{-1}[15]$, dentro de la cual la provincia del Cañar ubicada al sur, ocupa un importante lugar en la producción láctea, con un aporte promedio de $450.000 \mathrm{~L} \cdot \mathrm{d}^{-1}$, lo que representa el $6,76 \%$ de la producción nacional [16]. En este contexto, la ganadería bovina (Bos taurus) de producción lechera, es sin duda, uno de los sectores más importantes de la economía nacional, por la generación de empleo [27] y por su aporte del 13,41\% al Producto Interno Bruto (PIB) Agrícola y del 1,3\% al PIB Nacional [32].

El inicio de la ganadería lechera en Ecuador data desde hace 500 años (a); sin embargo, en el siglo XX hubo un avance significativo y progresivo que se mantiene hasta la actualidad [10]; no obstante, esta actividad está amenazada por factores como: el precio bajo de la leche, escasa presencia de programas de fomento ganadero [14], alta prevalencia de mastitis a nivel de finca [4], resistencia de los microorganismos a los antibióticos, entre ellos Staphylococcus aureus con el 65,63\% de resistencia a la Penicilina y Streptococcus agalactiae con el $56 \%$ a Penicilina y Cefalexina y el $52 \%$ a Cefalotina [33] entre otros.

Esta enfermedad es la más frecuente en sistemas de producción lechera [3], es un factor importante para el incremento del costo en la cria de ganado lechero [4], con gran impacto económico a nivel mundial, regional y local [21], existen factores que predisponen a los animales a sufrir la enfermedad entre ellos: raza, etapa de lactancia y prácticas de manejo [31]; de acuerdo a la visibilidad de signos clínicos se clasifica a la enfermedad en mastitis clínica o Mastitis Subclínica Bovina (MSB) [6, 26]. La forma de MSB causa mayores pérdidas económicas al provocar reducción en la producción y la calidad de la leche de los cuartos afectados sin poder ser detectada a tiempo [25], costos por tratamiento y sacrificio temprano de hembras lecheras con alto potencial genético, pérdidas durante el primer tercio de gestación tras desencadenar la liberación de mediadores inflamatorios como la prostaglandina, ocasionando lisis del cuerpo lúteo y consecuentemente pérdida de la gestación [22]; finalmente la enfermedad causa problemas en salud pública, sobre todo por el consumo de leche no pasteurizada [24].

Un estudio realizado por Bonifaz y Colango, en dos etapas y con intervalos de cuatro meses, en 42 fincas lecheras en Cayambe, Quito-Ecuador, reportó una prevalencia del $64 \%$ para la primera etapa y del $66 \%$ en la segunda etapa de investigación, y una incidencia del $70 \%$ de Mastitis Bovina (MB) [5] y como microorganismos en la presencia de esta patología se incluye a especies de Staphlococcus, Strepcococcus y bacterias Gram-negativas como: Klebsiella y coliformes, y como factores predisponentes se señala la falta de higiene en el proceso de ordeño, fallas en el equipo de ordeño y escaso uso de selladores [21]. En relación a lo anterior y por la importancia que tiene el tema, el presente estudio tuvo como objetivo detectar la presencia de MSB y factores asociados a la enfermedad en el cantón Biblián, provincia del Cañar-Ecuador.

\section{MATERIALES Y MÉTODOS}

\section{Área de estudio}

El estudio se desarrolló en el cantón Biblián (Cañar - Ecuador), ubicado a una altura promedio de 2.608 metros sobre el nivel del mar, con temperaturas que oscilan entre los 2 y $14^{\circ} \mathrm{C}$ y una pluviosidad anual con rangos de 750 a 1000 milímetros $\cdot \mathrm{a}^{-1}$.

En el estudio se emplearon un total de 1.440 cuartos mamarios, pertenecientes a 360 hembras (bovinas) en producción de leche entre el primer y quinto mes de lactancia y con 2 a 3 partos; la raza predominante en dicho estudio fue la Holstein Friesian y sus cruces, provenientes de 23 fincas del cantón Biblián, teniendo como principal recurso forrajero una mezcla entre Lolium perenne y Lolium.multiflorum (ray - grass), Poa pratensis (pasto azul), Trifolium repens y Trifolium pratense (trébol). De las 23 fincas estudiadas, 11 manejan ordeño mecánico (OMec) y las 12 restantes fincas con ordeño manual (OMan), y practican dos ordeños $\cdot \mathrm{d}^{-1}$ en horarios de cuatro de la mañana y cuatro de la tarde.

La toma de muestras de leche se realizó tras la limpieza con agua destilada tipo I, desinfección con alcohol etílico de 70 grados y secado de los pezones con toallas de papel; eliminando los primeros chorros de leche y empleando la paleta para el test de Mastitis California (CMT) se recolectó 2 mililitros $(\mathrm{mL})$ de leche por cada cuarto mamario y se adicionó $2 \mathrm{~mL}$ de reactivo de CMT, la mezcla se distribuyó de forma homogénea realizando movimientos circulares por 10 segundos (seg) [19]. La clasificación de la reacción creada entre el alkil-aril sulfonato de sodio y la cantidad de células somáticas (CS) presentes en la leche (gelificación), se realizó de acuerdo al método descrito por Smith en 1990, citado por Bedolla y col. [3], que arrojó su resultado cualitativo para cada cuarto. El contaje de células somáticas (CCS) se realizó empleando el analizador de CS Ekomilk Scan, marca Ekomilk, modelo Ultra Pro, serial: SN S00802306, Bulgaria, el recuento se realizó mediante un método recomendado por la norma NTE INEN 1529-5, Ecuador. La categorización de la escala de riesgo de infección se realizó de la siguiente manera: (+) 200.000 a $800.000 \mathrm{CS} \cdot \mathrm{mL}^{-1} \cdot$ vacas $^{-1}$ con bajo riesgo, y (++) 801.00 a 1.500 .000 $\mathrm{CS} \cdot \mathrm{mL}^{-1}$ animales de alto riesgo $(++)$; puesto que la norma INEN

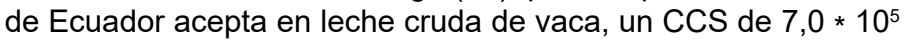
unidades formadoras de células [17].

\section{Análisis estadístico}

Se realizó un estudio observacional descriptivo, no probabilístico de conveniencia y corte transversal para determinar la prevalencia de MS en vacas de bajo y alto riesgo y su relación con ciertos factores asociados a la enfermedad: tipo de ordeño (mecánico o manual), aplicaciones de buenas prácticas antes y después del ordeño (lavado de ubre, secado individual y sellado de cuartos mamarios). La información fue organizada en tablas de contingencia y analizada mediante estadística descriptiva y una prueba de Ji-cuadrado para medir el grado de asociación entre las variables estudiadas.

El análisis y razón de prevalencia, odds ratio, valor $\mathrm{P}$ de la infección a nivel de cuartos mamarios se analizó a través del programa para análisis epidemiológico de datos EPIDAT versión 3.1 [34]; mientras que el cálculo de prevalencia de MS se determinó según la formula descrita por Rodríguez [26]. 


$$
\text { Prevalencia }=\left(\frac{\text { Número de vacas positivas }}{\text { Número de vacas muestreadas }}\right) \times 100
$$

\section{RESULTADOS Y DISCUSIÓN}

El análisis de resultados determinó 33 vacas que resultaron positivas a MSB, lo que determina una prevalencia de $9,1 \%$ (33/360), a nivel de cuartos mamarios del 4,03 \% (58/1440) y en fincas del 56,5\% (13/23). Los datos de prevalencia de MSB por cuartos mamarios son menores a los reportados por Bonifaz y Conlago [5], quienes señalan en su estudio, que el $64 \%$ de los cuartos mamarios evaluados estaban afectados con algún grado de mastitis, en vacas ubicadas en ganaderías de Cayambe, Pichincha-Ecuador. La prevalencia de MSB en las lecherías del sur de Ecuador según datos reportados por Murillo y col. [23] fue mayor en ganaderías medianas y menor en sistemas de producción de grandes y pequeños productores, encontrando que las actividades de pre y post ordeño influyeron negativamente en la presencia de MSB en las ganaderías, pequeñas, medianas y grandes de la provincia del Azuay.

Datos de prevalencia de MS en ganaderías lecheras de Colombia, $[2,6,7,29]$ señalan el $3,25 \%$ en vacas lactantes, el 34,4 y el $11,3 \%$ a nivel de cuartos mamarios positivos a CMT y el $43,4 \%$ en vacas, respectivamente, estos datos reflejan menor prevalencia a nivel de vacas y mayor en cuartos mamarios que los reportados en el presente estudio. En investigaciones realizadas en ganaderías de Perú se reportaron prevalencias de $72,3 \%$ en vacas y del $48,7 \%$ en cuartos mamarios considerando trazas como positivos [13]; mientras que Alvarado y col. [1] indicaron un porcentaje de prevalencia de $51 \%$ utilizando CMT y del $52 \%$ con el Test DCC (DeLaval Direct Cell Counter), los datos reportados superan el porcentaje de prevalencia de mastitis presente en vacas y cuartos mamarios registrados en Biblián, Ecuador. En Venezuela, en ganaderías productoras de leche, Ferraro y col. [11] y Castillo y col. [9] reportaron el 30,18 y 35,2 \%, según corresponde, siendo similar el porcentaje de prevalencia registrado en el presente estudio; sin embargo, difieren de los reportados en ganaderías lecheras de Cuba, García y col. [12] quienes reportan una prevalencia del $60 \%$ a nivel de rebaños durante los meses de abril y mayo.

Por otra parte, se clasificó a los cuartos mamarios de acuerdo al CCS, y se estableció parámetros de $200.000-800.000(+)$ como cuartos de bajo riesgo y de $801.000-1.500 .000(++)$ de alto riesgo, registrando 22 cuartos $(+)$ y 36 cuartos (++), lo que representa un $1,53 \%(22 / 1.440)$ y $2,5 \%(36 / 1440)$ de prevalencia, respectivamente.

La prevalencia de MS en cuartos de bajo riesgo $(+)$ con ordeño mecánico (OMec) fue de $2,87 \%$ (20/696), mientras que con oreño manual (OMan) de 0,27 \% (2/744). La aplicación de algunas prácticas de ordeño (lavado de ubre, secado y sellado de pezones) exhibieron prevalencias mayores; quizá, el resultado encontrado en este estudio, sea consecuencia de un mal manejo en lo que respecta al secado de la ubre, puesto que en la mayoría de sistemas con OMec no utilizan toalla individual para este fin. La razón de prevalencia dentro de las variables analizadas determinó que existe asociación entre el tipo de ordeño $(10,69 \%)[1,0 ; 2,0]$ y el secado individual $(9 \%)[0,25 ; 4,26]$ con la presencia de la enfermedad. El estudio de odds ratio obtenido para el tipo de ordeño $(10,98)[7,46 ; 10,19]$ y secado de la ubre $(9,17)$ indica un factor de riesgo para sufrir MS; mientras que el lavado $(0,194)$ y sellado de pezones $(0,255)$ son factores de prevención, encontrando diferencia significativa $(P<0,05)$ dentro de las variables analizadas, considerando que valores de OR mayores a 1 son factores de riesgo y valores menor a 1 se consideran como factores de protección, frente a la presentación de una enfermedad [18], TABLA I.

En cuartos mamarios de alto riesgo, la prevalencia fue de $3,01 \%(21 / 696)$ en OMec y $2,02 \%$ (15/744) en OMan; mientras que con la aplicación de prácticas de manejo (lavado de ubre, secado individual y sellado de pezones) la prevalencia de MSB en cuartos de alto riesgo tiende a ser menor, registrando valores de 2,$3 ; 0,7$ y $2,0 \%$, respectivamente. Las razones de prevalencia

TABLA I

Prevalencia y factores asociados en mastitis subclínica bovina de bajo riesgo (+)

\begin{tabular}{|c|c|c|c|c|c|c|c|}
\hline \multirow[b]{2}{*}{ Variable } & \multirow[b]{2}{*}{ Referencia } & \multicolumn{6}{|c|}{ Mastitis Subclínica } \\
\hline & & $\begin{array}{c}\text { Prevalencia } \\
\%\end{array}$ & $\begin{array}{c}\text { Razón de } \\
\text { prevalencia } \\
\text { IC ( } 95 \%)\end{array}$ & $\begin{array}{l}\text { Prevalencia de } \\
\text { exposición } \\
\text { IC ( } 95 \%)\end{array}$ & $\begin{array}{c}\text { Razón de } \\
\text { prevalencia } \\
\text { de exposición } \\
\text { IC ( } 95 \%)\end{array}$ & Valor P & $\begin{array}{c}\text { Odds Ratio } \\
\text { IC ( } 95 \%)\end{array}$ \\
\hline \multirow{2}{*}{ Ordeño } & Mecánico & 2,87 & \multirow{2}{*}{10,69} & Enfermos (91) & \multirow{2}{*}{1,9} & \multirow{2}{*}{0,0001} & \multirow{2}{*}{10,98} \\
\hline & Manual & 0,27 & & No enfermos (48) & & & \\
\hline \multirow{2}{*}{ Lavado de ubre } & No & 0,41 & \multirow{2}{*}{0,2} & Enfermos (9) & \multirow{2}{*}{0,27} & \multirow{2}{*}{0,0141} & \multirow{2}{*}{0,194} \\
\hline & $\mathrm{Si}$ & 2,1 & & No enfermos (34) & & & \\
\hline \multirow{2}{*}{ Secado individual } & No & 2,1 & \multirow{2}{*}{9} & Enfermos (95) & \multirow{2}{*}{1,37} & \multirow{2}{*}{0,0087} & \multirow{2}{*}{9,1702} \\
\hline & $\mathrm{Si}$ & 0,2 & & No enfermos (70) & & & \\
\hline \multirow{2}{*}{ Uso de sellado } & NO & 0,6 & \multirow{2}{*}{0,26} & Enfermos (18) & \multirow{2}{*}{0,39} & \multirow{2}{*}{0,0081} & \multirow{2}{*}{0,255} \\
\hline & SI & 2,3 & & No enfermos (47) & & & \\
\hline
\end{tabular}


Detección y factores asociados a Mastitis Subclínica Bovina / Cuenca-Condoy y col.

encontradas en cuartos de alto riesgo determinaron asociación entre el secado de la ubre y la prevalencia de mastitis $(4,7 \%)$ $[0,23 ; 1,83]$. Los valores encontrados en el estudio de odds ratio refieren que todas las variables (tipo de ordeño, lavado de ubre, secado y sellado de pezones) representan un factor de riesgo a sufrir la enfermedad, siendo mayor para el secado $(4,84)[4,15$; $7,27]$, cuando no se lo realiza con toalla individual para cada animal, encontrando diferencia significativa $(P=0,0041)$ dentro de esta variable, TABLA II.

En este estudio se determinó, que existe asociación entre el tipo de ordeño y la presencia de MS en cuartos de alto y bajo riesgo, siendo mayor en sistemas lecheros con OMec que con OMan; los datos coinciden con los reportados por Ruiz y col. [28], quienes indican que los rebaños ordeñados mecánicamente tuvieron mayor prevalencia de MS, señalando datos de prevalencia con CMT de 39,3 y $54,8 \%$ y con CCS de 33,4 y $49,4 \%$ para OMan y OMec, respectivamente; así mismo, los datos fueron corroborados por Calvinho y Tirante [8] quienes refieren que de un total de 6.420 cuartos mamarios que fueron ordeñados de forma mecánica OMec, el $53 \%$ resultó positivo a MS, y de 1.580 cuartos mamarios ordeñados de forma manual OMan el 35,2 \% presentó la enfermedad en su forma subclínica.

Por otra parte, en esta investigación se encontró que el OMec incrementa el riesgo de padecer la enfermedad, quizá porque el equipo y sistema de ordeño no se ejecutan correctamente; a esto se añade que el secado de pezones en fincas con OMec no lo realizan con toalla individual, lo que podría dar lugar a incrementar la presencia de MSB; los datos coinciden con los reportados por Mendoza y col. [20], quienes señalan que la ausencia de prácticas de manejo incrementa el riesgo de las vacas a padecer la enfermedad e incrementar los valores de prevalencia; por su parte Santivañez y col. [30] registraron en su estudio, un odds ratio de 2,1 para la ausencia de la higiene de manos antes del ordeño, determinando un riesgo a padecer la enfermedad de 2,1 veces más en sistemas lecheros donde no se realiza esta actividad.

\section{CONCLUSIÓN}

La prevalencia de MSB encontrada en la provincia de Cañar, cantón Biblián-Ecuador fue de $9,1 \%$ en vacas, $4,03 \%$ en cuartos mamarios y $56,5 \%$ en fincas lecheras y como factores asociados a MSB se determinó que el OMec y el secado de pezones con toalla colectiva en vacas lecheras, incrementan el riesgo de padecer la enfermedad.

\section{AGRADECIMIENTOS}

Los autores agradecen al Ministerio de Agricultura y Ganadería del Cañar y al Gobierno Autónomo Descentralizado de la parroquia Nazón, cantón Biblián por el apoyo brindado con la organización de los productores durante el desarrollo de la fase de muestreo.

\section{REFERENCIAS BIBLIOGRÁFICAS}

[1] ALVARADO, W.; GONZÁLEZ, J.; QUILCATE, C.; SAUCEDO, J.; BARDALES, J. Factores de prevalencia de mastitis subclínica en vacas lecheras del distrito de Florida, Región Amazonas, Perú. Rev. Inv. Vet. Perú. 30(2): 923-931. 2019.

[2] ANDRADE, R.; CARO, Z.; DALLOS, A. Prevalencia de mastitis subclínica bovina y su etiología infecciosa en fincas lecheras del Altiplano Boyacense (Colombia). Rev. Científ. FCV-LUZ. XXIV(4): 305-310. 2014.

[3] BEDOLLA, C.; CASTAÑEDA, V. Métodos de detección de mastitis bovina. REDVET. VIII(9): 1-17.2007.

[4] BITTAR, A.; OLIVEIRA, T.; MIZUKI, T.; MARTINI, K.; MELLO, L.; VIEIRA DA SILVA, A.; ALMEIDA, L. Prevalência e etiologia da mastite bovina na região de Nova Tebas, Paraná. Semina: Cien. Agrár. 35(2): 835-844. 2014.

[5] BONIFAZ, N.; CONLAGO, F. Prevalencia e incidencia de mastitis bovina mediante la prueba de california mastitis test con identificación del agente etiológico,en Paquiestancia, Ecuador. La Granja. 24(2): 1-16. 2016.

TABLA II

Prevalencia y factores asociados en mastitis subclínica bovina de alto riesgo (++)

\begin{tabular}{|c|c|c|c|c|c|c|c|}
\hline \multirow[b]{2}{*}{ Variable } & \multirow[b]{2}{*}{ Referencia } & \multicolumn{6}{|c|}{ Mastitis Clínica } \\
\hline & & $\begin{array}{c}\text { Prevalencia } \\
\%\end{array}$ & $\begin{array}{c}\text { Razón de } \\
\text { prevalencia } \\
\text { IC ( 95\%) }\end{array}$ & $\begin{array}{l}\text { Prevalencia de } \\
\text { exposición } \\
\text { IC ( 95\%) }\end{array}$ & $\begin{array}{c}\text { Razón de } \\
\text { prevalencia } \\
\text { de exposición } \\
\text { IC ( } 95 \%)\end{array}$ & Valor P & $\begin{array}{c}\text { Odds Ratio } \\
\text { IC ( } 95 \%)\end{array}$ \\
\hline \multirow{2}{*}{ Ordeño } & Mecánico & 3,01 & \multirow{2}{*}{1,5} & Enfermos (58) & \multirow{2}{*}{1,25} & \multirow{2}{*}{0,224} & \multirow{2}{*}{1,512} \\
\hline & Manual & 2,02 & & No enfermos (48) & & & \\
\hline \multirow{2}{*}{ Lavado de ubre } & No & 3,10 & \multirow{2}{*}{1,4} & Enfermos (42) & \multirow{2}{*}{1,25} & \multirow{2}{*}{0,3001} & \multirow{2}{*}{1,424} \\
\hline & $\mathrm{Si}$ & 2,20 & & No enfermos (33) & & & \\
\hline \multirow{2}{*}{ Secado individual } & No & 3,30 & \multirow{2}{*}{4,7} & Enfermos (92) & \multirow{2}{*}{1,32} & \multirow{2}{*}{0,0041} & \multirow{2}{*}{4,840} \\
\hline & $\mathrm{Si}$ & 0,70 & & No enfermos (69) & & & \\
\hline \multirow{2}{*}{ Uso de sellado } & NO & 3,20 & \multirow{2}{*}{1,6} & Enfermos (58) & \multirow{2}{*}{1,27} & \multirow{2}{*}{0,1363} & \multirow{2}{*}{1,657} \\
\hline & SI & 1,90 & & No enfermos (46) & & & \\
\hline
\end{tabular}


[6] CALDERÓN, A.; RODRÍGUEZ, V. Prevalencia de mastitis bovina y su etiología infecciosa en sistemas especializados en producción de leche en el altiplano cundiboyacense (Colombia). Rev. Colomb. Cien. Pec. 21: 582-589. 2008.

[7] CALDERÓN, A.; RODRÍGUEZ, V.; ARRIETA, G.; MÁTTAR, S. Prevalencia de mastitis bovina en sistemas doble propósito en Montería (Colombia): etiología y susceptibilidad antibacteriana. Rev. Colomb. Cien. Pec. 24: 19-28. 2011.

[8] CALVINHO, L.; TIRANTE, L. Prevalencia de microorganismos patógenos de mastitis bovina y evolución del estado de salud de la glándula mamaria en argentina en los últimos 25 años. FAVE Cien. Vet. 4(1): 30-40. 2005.

[9] CASTILLO, M.; SUNIAGA, J.; ROJAS, G.; HERNÁNDEZ, J.; CAAMAÑO, J; URBINA, A.; TOVAR, L. Estudio de la prevalencia de mastitis subclínica en la zona alta del estado de Mérida. Agricult. Andin. 16: 39 - 48. 2009.

[10] CENTRO DE LA INDUSTRIA LÁCTEA DEL ECUADOR. La leche del Ecuador, historia de la lecheria ecuatoriana, Pichincha, Quito-Ecuador. 2015. En línea: https://bit. ly/3AwhZoa. 20-03-2021.

[11] FERRARO, L.; SCARAMELLI, A.; TROYA, H. Prevalencia de la mastitis subclínica bovina en Venezuela y evaluación de la prueba de mastitis de California (CMT) como prueba diagnóstica. Rev. Científ. FCV-LUZ. IX(2): 81-90. 1999.

[12] GARCÍA, F.; SÁNCHEZ, T.; LÓPEZ, O.; BENÍTEZ, M. Prevalencia de mastitis subclínica y microorganismos asociados a esta. Pastos y Forrajes. 41(1): 5-40. 2018.

[13] GÓMEZ, O.; SANTIVAÑEZ, C.; ARAUCO, F.; ESPEZUA, O.; MANRIQUE, J. Criterios de interpretación para California Mastitis Test en el diagnóstico de mastitis subclínica en bovinos. Rev. Inv. Vet. Perú. 26(1): 86-95. 2015.

[14] HARO, R. Informe de recursos zoogéneticos de Ecuador. QuitoEcuador. 2003. En línea: https://bit.ly/2V9IS28. 12-03-2021

[15] INSTITUTO NACIONAL DE ESTADÍSTICAY CENSOS (INEC). Censo Agropecuario.2019. En línea: https://bit.ly/3hMtnUw. 12-03-2021.

[16] INSTITUTO NACIONAL DE ESTADÍSTICA Y CENSOS (INEC). Encuesta de superficie y producción agropecuaria continua(ESPAC). Estadísticas Agropecuarias, QuitoEcuador. 2019. En línea: https://bit.ly/3hMtnUw. 20-03-2021.

[17] INSTITUTO ECUATORIANO DE NORMALIZACIÓN (INEN). Norma técnica ecuatoriana NTE INEN 9:2012. Requisitos microbiológicos de la leche cruda tomada en hato. QuitoEcuador. 2012. En línea: https://bit.ly/3AHuCgu. 23-03-2021.

[18] MARTIN, S.; MEEK, A.; WILLEBERG, P. Epidemiologic Measures of Association. In: Veterinary Epidemiology: Principles and Methods. Low State University Press (Ed.). 1st. Ed. Pp 130-134. 1987.

[19] MELLENBERG, R.; ROTH, C. Hoja de Información de la Prueba de Mastitis California (CMT). 2004. En línea: https:// bit.ly/3hNCVym. 22/06/2021.
[20] MENDOZA, J.; VERA, Y.; PEÑA, L. Prevalencia de mastitis subclínica, microorganismos asociados y factores de riesgo identificados en hatos de la provincia de Pamplona, Norte de Santander. Rev. Med. Vet. Zoot. 64(2): 11-24. 2017.

[21] MERA, R.; MUÑOZ, M.; ARTIEDA, J.; ORTÍZ, P.; GONZÁLEZ, R.; VEGA, V. Mastitis bovina y su repercusión en la calidad de la leche. REDVET. 18(11): 1-16. 2017.

[22] MIRANDA, S.; ALBUJA, C.; TRÍBULO, H. Asociación entre la mastitis subclínica con la pérdida temprana de gestación en un hato de vacas lecheras. La Granja. 30(2): 48-56. 2019.

[23] MURILLO, Y.; VÁZQUEZ, J.; AYALA, L.; PESÁNTEZ, M.; PESÁNTEZ, J.; SERPA, V.; SAMANIEGO, J. La rutina de ordeño en la prevalencia de la mastitis subclínica en lecherías del sur del Ecuador. MASKANA. Producción Animal. 8: 41-43. 2017.

[24] MURPHY, S.; MARTIN, N.; BARBANO, D.; WIEDMANN, M. Influence of raw milk quality on processed dairy products: How do raw milk quality test results relate to product quality and yield? J. Dairy Sci. 99(12): 10128-10149. 2016.

[25] OLIVEIRA, C.; SOUSA, M.; SILVA, N.; MENDONÇA, C.; SILVEIRA, J.; OAIGEN, R.; ANDRADE, S.; BARBOSA, J. Prevalência e etiologia da mastite bovina na bacia leiteira de Rondon do Pará, estado do Pará. Pesq. Vet. Bras. 31(2): 104-110. 2011.

[26] RODRÍGUEZ, L. Prevalencia de mastitis subclínica en tambo lechero en Paraguay. Rev. Med. Vet. 1(40): 61-68. 2020.

[27] RUIZ, P. La importancia de la producción de leche en el Ecuador. Reforma Agraria. Ed. SIPAE. Pp. 35-41. 2006.

[28] RUIZ, P.; GOMES, G.; MOTA, R.; SAMPAIO, E.; LUCENA, E.; BENONE, S. Prevalencia de Mastitis Bovina Subclínica y Microorganismos Asociado: Comparación entre Ordeño Manual y Mecánico, en Pernambuco, Brasil. Rev. Salud Anim. 1: 57-64. 2011.

[29] SÁNCHEZ, M.; GUTIÉRREZ, N.; POSADA, I. Prevalencia de mastitis bovina en el Cañón de Anaime, región lechera de Colombia, incluyendo etiología y resistencia antimicrobiana. Rev. Inv. Vet. Perú. 29(1): 226-239.2018.

[30] SANTIVAÑEZ, C.; GÓMEZ, O.; CÁRDENAS, L.; ESCOBEDO, M.; BUSTINZA, R.; PEÑA, J. Prevalencia y factores asociados a la mastitis subclínica bovina en los Andes peruanos. Vet. Zoot. 7(2): 92-104. 2013.

[31] SINHA, M.; THOMBARE, N.; MONDAL, B. Subclinical Mastitis in Dairy Animals: Incidence, Economics, and Predisposing Factors. Sci. World J. 15(1): 1-4. 2014.

[32] TERÁN, J. Análisis del mercado de la leche en Ecuador: factores determinantes y desafíos. 2019. Universidad Politécnica de Valencia. En línea: https://bit.ly/3hJe9Q0.18-03-2021

[33] VILLANUEVA, G; MORALES, S. Resistencia antibiótica de patógenos bacterianos aislados de mastitis clínica en bovinos de crianza intensiva. REDVET. 18(12): 1-13. 2017.

[34] XUNTA DE GALICIA, EPIDAT v 3.1. Santiago de Compostela, A Coruña-España. 2006. 
